\title{
Trousseau's syndrome caused by bladder cancer producing granulocyte colony-stimulating factor and parathyroid hormone-related protein: A case report
}

\author{
TOMONORI KATO ${ }^{1}$, KENJI YASUDA ${ }^{1}$, HIROAKI IIDA ${ }^{1}$, AKIHIKO WATANABE $^{1}$, \\ YASUYOSHI FUJIUCHI ${ }^{1}$, SHIGEHARU MIWA ${ }^{2}$, JOHJI IMURA ${ }^{2}$ and AKIRA KOMIYA ${ }^{1}$ \\ Departments of ${ }^{1}$ Urology and ${ }^{2}$ Diagnostic Pathology, \\ Graduate School of Medicine and Pharmaceutical Sciences for Research, University of Toyama, Toyama 930-0194, Japan
}

Received March 31, 2015; Accepted June 2, 2016

DOI: $10.3892 / \mathrm{ol} .2016 .5152$

\begin{abstract}
The present study reports a rare case of bladder cancer that caused Trousseau's syndrome, a malignancy-associated hypercoagulative state leading to venous or arterial thrombosis. A 38-year-old Japanese female patient experienced a sudden onset of visual field defects and lower limb weakness. Cerebral magnetic resonance imaging revealed multi-regional hypointense areas compatible with acute cerebral infarction. Despite the repeated administration of anticoagulant treatment with heparin, the condition could not be adequately controlled and thromboembolic events occasionally recurred. Several tumor markers, including carbohydrate antigen 19-9, cancer antigen 125 , carcinoembryonic antigen, cytokeratin 19 fragment and squamous cell carcinoma antigen levels, were elevated. Consequently, computed tomography scans were performed, which revealed a massive bladder tumor with multiple bone and lymph node metastases. The patient also exhibited other paraneoplastic disorders, including leukocytosis due to granulocyte colony-stimulating factor (G-CSF) production, and hypercalcemia due to parathyroid hormone-related protein (PTHrP) production. Transurethral resection of the bladder tumor was performed, and the tumor was pathologically confirmed as urothelial cell carcinoma. Immunohistochemical testing revealed positive staining for G-CSF and PTHrP. Despite undergoing gemcitabine/cisplatin-based systemic chemotherapy, the
\end{abstract}

Correspondence to: Dr Tomonori Kato, Department of Urology, Graduate School of Medicine and Pharmaceutical Sciences for Research, University of Toyama, 2630 Sugitani, Toyama 930-0194, Japan

E-mail: jpn3899kato@yahoo.co.jp

Abbreviations: G-CSF, granulocyte colony-stimulating factor; $\mathrm{PTHrP}$, parathyroid hormone-related protein

Key words: Trousseau's syndrome, bladder cancer, granulocyte colony-stimulating factor, parathyroid hormone-related protein, cerebral infarction disease developed rapidly and the patient succumbed to the disease within 3 months of initial symptoms. The present case indicates that occult visceral malignancy should be considered in patients with unexplained thromboembolism.

\section{Introduction}

Trousseau's syndrome is a well-known malignancy-associated hypercoagulative state, which leads to venous or arterial thrombosis (1). Since its original description in 1865 (1), numerous studies have confirmed the common association between cancer and thromboembolism. Approximately $15 \%$ of cancer patients suffer a thromboembolic event during their clinical course, and as many as 50\% exhibit evidence of venous thromboembolism on postmortem examinations (2). Trousseau's syndrome occasionally occurs in patients with carcinomas of the pancreas, ovaries or lungs (3). Trousseau syndrome is usually diagnosed clinically as no specific biochemical markers exist for this syndrome. With regard to the treatment of Trousseau syndrome, it is important to control the causative tumor. Adequate anti-coagulant therapies are also required for treatment (4). At present, Trousseau syndrome has a poor prognosis as controlling thrombosis remains difficult (5). To the best of our knowledge, the present study is the first reported case of Trousseau's syndrome occurring in a patient with bladder cancer. The patient also presented with other paraneoplastic disorders, including leukocytosis caused by granulocyte colony-stimulating factor (G-CSF) production, and hypercalcemia caused by parathyroid hormone-related protein (PTHrP) production. Leukocytosis and hypercalcemia are paraneoplastic syndromes associated with high-grade aggressive tumors $(6,7)$. In addition, the present report discusses paraneoplastic syndrome, including leukocytosis and hypercalcemia. This study was approved by the ethics committee of Toyama University Hospital (Toyama, Japan).

\section{Case report}

A 38-year-old Japanese female patient was admitted to Toyama University Hospital in March 2014, due to a sudden 
onset of visual field defects and lower limb weakness from an unknown cause. She had a history of surgical procedures for spina bifida and hydrocephalus following birth; this had led to neurogenic bladder in the patient, requiring intermittent catheterization for 30 years. Cerebral magnetic resonance imaging revealed multi-regional hypointense areas compatible with acute cerebral infarction, in addition to the known hydrocephalus. At the time of admission, laboratory examination results revealed a high platelet count $\left(41.6 \times 10^{4} / \mu 1\right.$; normal range, $\left.16.3-34 \times 10^{4} / \mu \mathrm{l}\right)$ and elevated fibrinogen level $(611 \mathrm{mg} / \mathrm{dl}$; normal range, 200-400 mg/dl). Other coagulation study results were within the normal ranges: D-dimer, $1.0 \mu \mathrm{g} / \mathrm{ml}$ (normal range, $0-1.0 \mu \mathrm{g} / \mathrm{ml}$ ); prothrombin time, $12.3 \mathrm{sec}$ (normal range, 11.8-14.2 sec); activated partial thromboplastin time, $35.0 \mathrm{sec}$ (normal range, 26.5-41.8 sec); antithrombin III activity, 104\% (normal range, 80-120\%). Anticoagulation therapy with low molecular weight heparin was initiated immediately; however, despite continuous antithrombotic therapy, repeated episodes of cerebral infarction occurred. Venous Doppler ultrasonography revealed no thrombi formation in the bilateral lower limbs, and Holter electrocardiogram showed no signs of atrial fibrillation. Moyamoya disease, collagenosis, sarcoidosis, vasculitis and coagulation in response to infection were suspected as potential underlying diseases, but no etiology could be determined. Trousseau's syndrome was also considered in the differential diagnosis, and several tumor markers were measured, several of which were found to be elevated, as described below. Consequently, computed tomography scans were performed, revealing a massive bladder tumor, multiple bone metastases (Th 9 spine, right coxal bone and left proximal femur), multiple enlarged lymph nodes in the retroperitoneum and pelvis, and bilateral hydronephrosis (Fig. 1).

The levels of serum albumin had decreased to $2.0 \mathrm{~g} / \mathrm{dl}$ (normal range, 3.8-5.0 g/dl), while those of alkaline phosphatase had increased to $581 \mathrm{IU} / 1$ (normal range, 100-325 IU/1). C-reactive protein was $15.2 \mathrm{mg} / \mathrm{dl}$ (normal value, $<0.3 \mathrm{mg} / \mathrm{dl}$ ). Laboratory data also indicated marked leukocytosis and hypercalcemia [white blood cell count, 107,000/ $\mathrm{mm}^{3}$ (neutrophils, 91.5\% ; lymphocytes, $2.0 \%$ ) (normal range, 3,300-9,000/ $\mathrm{mm}^{3}$ ); serum calcium, $14.4 \mathrm{mg} / \mathrm{dl}$ (normal range, $8.5-10.5 \mathrm{mg} / \mathrm{dl}$ )] The serum levels of G-CSF and PTHrP were found to be elevated [G-CSF, $77.1 \mathrm{pg} / \mathrm{ml}$ (normal range, 0-39.0 pg/ml); PTHrP, $11.1 \mathrm{pmol} / 1$ (normal value, <1.1 pmol/l)]. Controlling the calcium level was challenging, despite the administration of bisphosphonates and calcitonin.

The following tumor markers were found to be elevated: Serum carbohydrate antigen (CA)19-9, $63 \mathrm{U} / \mathrm{ml}$ (normal value, $<37 \mathrm{U} / \mathrm{ml}$ ); CA125, $376 \mathrm{U} / \mathrm{ml}$ (normal value, <35 U/ml); carcinoembryonic antigen, $6.3 \mathrm{ng} / \mathrm{ml}$ (normal range, 0-3.4 ng/ml); cytokeratin 19 fragment, $12.0 \mathrm{ng} / \mathrm{ml}$ (normal range, $0-2.8 \mathrm{ng} / \mathrm{ml}$ ); squamous cell carcinoma (SCC) antigen, $95 \mathrm{ng} / \mathrm{ml}$ (normal range, $0-1.5 \mathrm{ng} / \mathrm{ml}$ ). The levels of $\alpha$-fetoprotein, neuron-specific enolase and pro-gastrin-releasing peptide levels were within the normal limits.

Subsequently, transurethral resection of the bladder tumor was performed. According to cystoscopic findings, the tumor appeared to be a predominantly non-papillary submucosal tumor with a partially papillary appearance. The lumen area had become constricted by the submucosal tumor, which occupied most of the inner surface of the bladder (Fig. 2).
$\mathbf{A}$

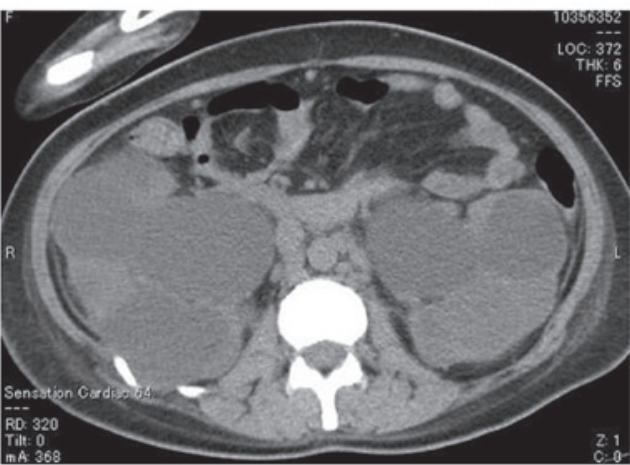

B

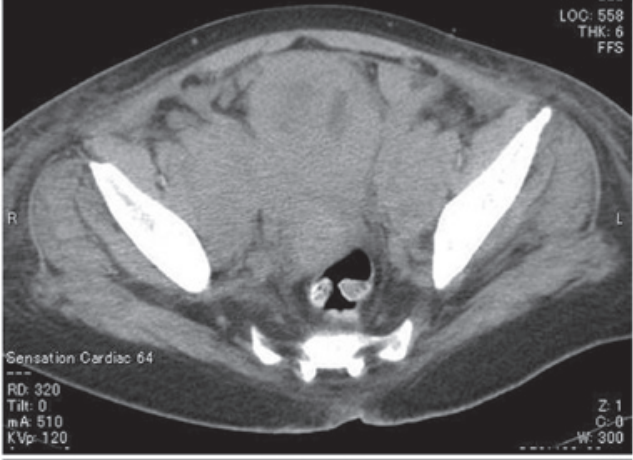

C

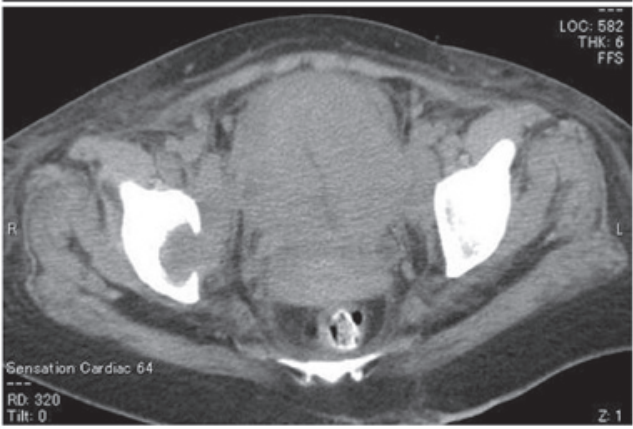

Figure 1. Computed tomography scans revealed a massive bladder tumor, multiple bone metastases and multiple enlarged lymph nodes in the retroperitoneum and pelvis, as well as bilateral hydronephrosis. (A) Bilateral hydronephrosis was observed. (B) Multiple lymph node metastases were observed inside the pelvis. (C) A massive bladder tumor was detected, and right coxal bone metastasis was also observed.

Serial sections of $4 \mu \mathrm{m}$ were cut from formalin-fixed, paraffin-embedded blocks of resected tissue. Pathological examination findings from the resected specimens revealed necrotic, invasive, poorly-differentiated urothelial carcinoma (Fig. 3A and C). For immunohistochemical analysis, sections were deparaffinized in xylene, rehydrated through decreasing grades of alcohol to water, and subjected to heat in target retrieval buffered solution (Dako Japan Co., Ltd., Tokyo, Japan) in a microwave oven for $10 \mathrm{~min}$. Endogenous peroxidase activity was quenched in $3 \%$ hydrogen peroxide solution in distilled water and sections were incubated with the following primary antibodies in a wet chamber and irradiated intermittently for $15 \mathrm{~min}$ : Mouse monoclonal anti-granulocyte-colony stimulating factor (1:100; Immuno-Biological Laboratories Co., Ltd., Gunma, Japan) and rabbit polyclonal PTHrP (1:50; Atlas Antibodies, Stockholm, Sweden). After rinsing in Tris-buffered saline (TBS) containing $0.1 \%$ Tween 20, the sections were incubated with Envision+/horseradish peroxidase (Dako Japan Co., Ltd.), and irradiated intermittently for $15 \mathrm{~min}$. After rinsing in TBS, 3,3'-diaminobenzidine was 


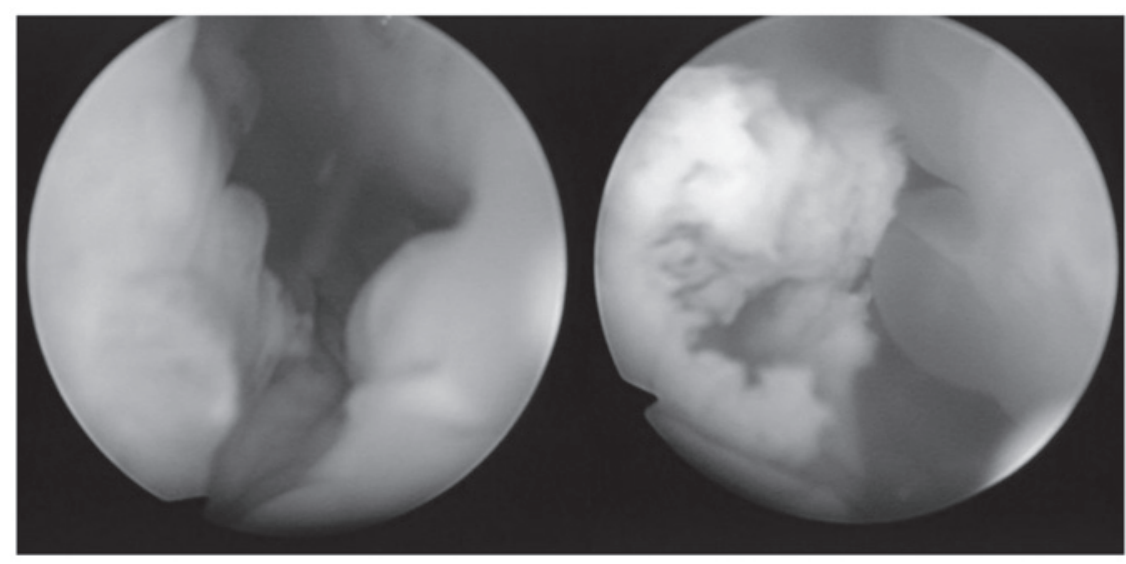

Figure 2. Cystoscopic findings. The tumor appeared to be a predominantly non-papillary submucosal tumor (left) with a partially papillary appearance (right). The lumen area had become constricted by the submucosal tumor, which occupied most of the inner surface of the bladder.
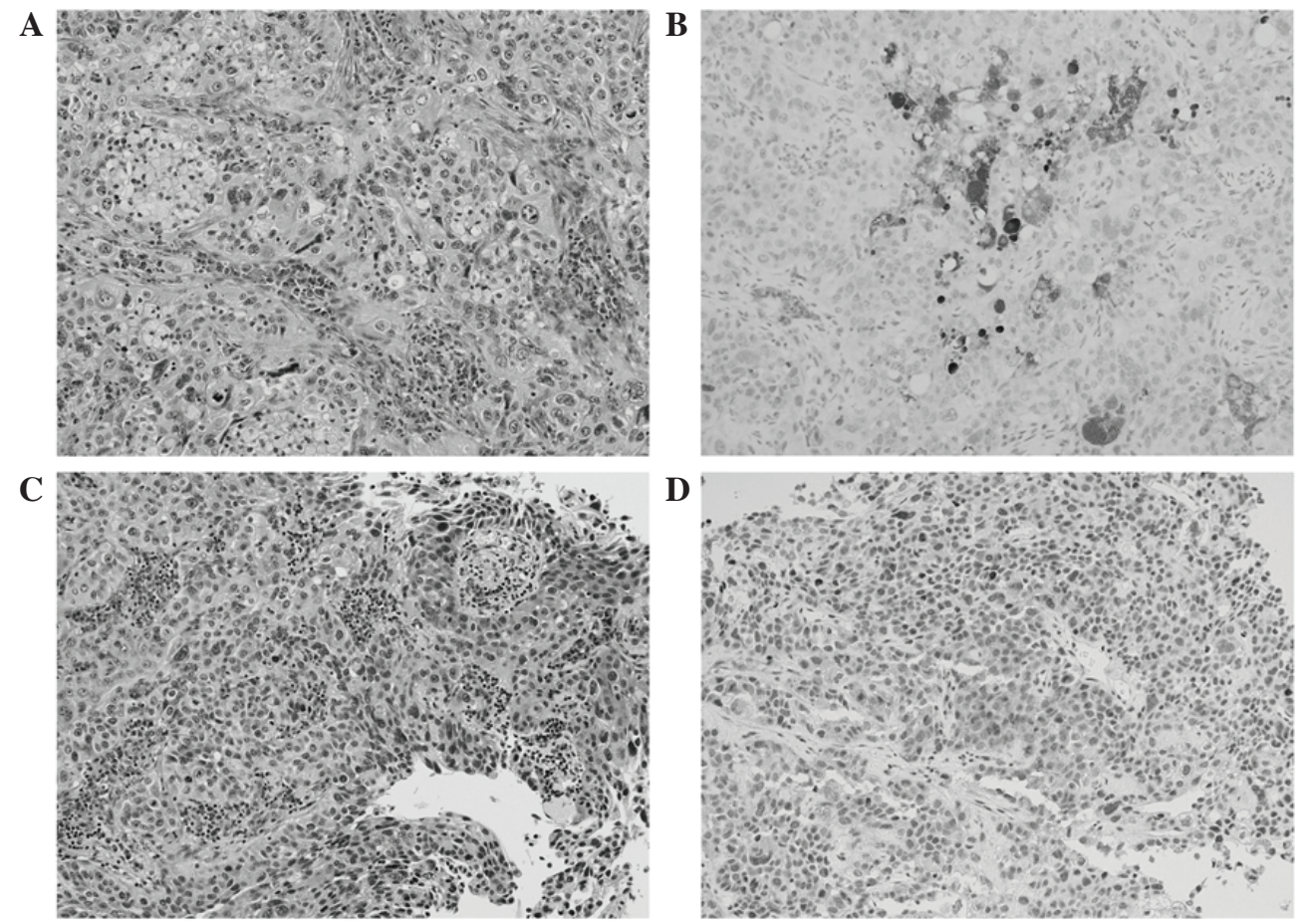

Figure 3. (A and C) Hematoxylin \& eosin staining of the tumor specimen showed poorly-differentiated urothelial carcinoma. Immunohistochemical staining results revealed (B) focally or diffusely positive reactivity for granulocyte colony-stimulating factor, and (D) scattered strong immunoreactivity for parathyroid hormone-related protein. Magnification, x200.

applied as a substrate for the secondary antibody. Finally, sections were counterstained with hematoxylin for $1 \mathrm{~min}$, dehydrated through graded alcohols, and cleared in xylene. Omission of the primary antibody was used as a negative control. Immunohistochemical testing revealed positive staining for G-CSF and PTHrP. Fig. 3B shows immunohistochemical staining for G-CSF, which was focally or diffusely positive. Scattered strong immunoreactivity was observed with PTHrP (Fig. 3D).

The disease developed rapidly and the patient's consciousness deteriorated, presumably due to severe hypercalcemia. Subsequently, gemcitabine/cisplatin-based systemic chemotherapy was administered. Although temporary, the patient's serum calcium level decreased to $8.0 \mathrm{mg} / \mathrm{dl}$ and the white blood cell count to $1,160 / \mathrm{mm}^{3}$, and she seemed to regain her consciousness following chemotherapy; however, 9 days from the initiation of chemotherapy, the patient developed sudden oculogyric crisis due to hemorrhagic cerebral infarction. Heparin therapy was inevitably terminated to control cerebral hemorrhage and, thereafter, her neurological disturbance advanced rapidly. The patient succumbed to respiratory failure due to brainstem infarction 15 days from the initiation of chemotherapy.

\section{Discussion}

Trousseau's syndrome is a well-known paraneoplastic synd rome in which thromboembolism is caused by a malignancy; it was 
first reported by Armand Trousseau in 1865 (1). Although no standard definition of the syndrome has been established, certain scientists have defined it as 'hypercoagulability syndrome associated with cancer' or 'malignancy-related thromboembolism' $(1,2)$. Trousseau's syndrome occasionally occurs in patients with carcinomas of the pancreas, ovaries or lungs (3); however, to the best of our knowledge, this is the first reported case of Trousseau's syndrome occurring in a patient with bladder cancer. It may have been overlooked partly due to the lack of biochemical markers for this syndrome and partly due to the fact that it has not yet been conclusively defined. The development of a novel marker to diagnose Trousseau's syndrome is required.

Several mechanisms have been proposed to explain the occurrence of hypercoagulation in patients with malignancy, including thromboplastin-like substances, fibrin deposition, direct factor $\mathrm{X}$ activation by tumor proteases, tissue factor, cysteine protease, tumor hypoxia, tumor-induced inflammatory cytokines and carcinoma-associated mucins (1,8-10). Among them, mucins are thought to trigger this syndrome $(9,10)$. The serum tumor markers CA19-9 (also known as MUC-1/Y), CA125 (also known as MUC-16) and CA15-3 (also known as MUC-1) are well known tumor-associated mucins; these are large, heavily glycosylated molecules that are resistant to denaturation, boiling and proteases (11). Carcinoma-associated mucins are thought to induce platelet-rich microthrombi with increasing tissue factor and selectins, resulting in intravascular hypercoagulation $(1,12)$. Indeed, Trousseau's syndrome is occasionally associated with mucin-producing adenocarcinoma $(1,9,13)$. Although the present case lacks the typical findings of mucin-producing carcinoma, such as intracytoplasmic mucin or extracellular mucin pools, the serum tumor markers CA19-9 and CA125 were found to be markedly elevated.

The expression CA125 and CA15-3 is believed to be correlated with the incidence of thromboembolism in cancer patients $(4,14)$. In the present case, in addition to the elevated CA125 levels, the elevated CA19-9 levels may also have been involved in the formation of thromboembolism. In undiagnosed patients with occult cancer who develop thromboembolism, mucin tumor markers may indicate the origin of mucin-producing carcinoma.

Controlling the causative tumor and providing immediate systemic anticoagulation is crucial for the treatment of Trousseau's syndrome (15). Although there is no established evidence regarding the effects of anticoagulant treatment on Trousseau's syndrome, heparin appears to be more effective than vitamin $\mathrm{K}$ antagonists $(1,3,16)$. In addition to the activation of antithrombin, heparin cofactor II and protein $\mathrm{C}$ inhibitor, heparin interrupts the binding of selectins, which mediates tumor-mucin interactions (1). In the present case, even though anticoagulant therapies with heparin were performed, the thromboembolic events could not be adequately controlled and mortality could not be avoided. In addition to the use of anticoagulation, it is also very important to treat the underlying malignancy.

Leukocytosis and hypercalcemia are paraneoplastic syndromes associated with high-grade aggressive tumors. Hypercalcemia associated with bladder cancer is not uncommon. Humoral hypercalcemia of malignancy, first described by Albright in 1941 (6), is the most common paraneoplastic syndrome and is caused by excessive production of
PTHrP, which is released from the tumor cells. PTHrP, whose amino-terminal is identical to that of parathyroid hormone, binds to the parathyroid hormone receptor and may stimulate osteoclastic bone resorption and produce hypercalcemia (7).

Certain aggressive tumors have been reported to produce G-CSF, resulting in marked leukocytosis without any infection $(17,18)$. G-CSF is a hematopoietic growth factor that stimulates granulocyte proliferation and differentiation (17). Leukocytosis in association with bladder cancer is relatively rare, with $\sim 35$ cases of leukocytosis in bladder cancer due to G-CSF production reported in the literature. G-CSF-producing bladder cancer has a poor prognosis (17).

A few bladder tumors simultaneously producing G-CSF and PTHrP have been reported (18). The majority of tumors producing G-CSF and PTHrP are SCCs, possibly due to the fact that they are both produced by keratinocytes. Although the present case lacked the typical findings of SCC in the resected specimen, the patient's serum SCC antigen was markedly elevated.

In summary, the present study reported the rare case of a patient presenting with various paraneoplastic syndromes, including Trousseau's syndrome, which were associated with a bladder cancer. Occult visceral malignancy should be considered in patients with unexplained thromboembolism.

\section{References}

1. Varki A: Trousseau's syndrome: Multiple definitions and multiple mechanisms. Blood 110: 1723-1729, 2007.

2. Bick RL: Cancer-associated thrombosis. N Engl J Med 349: 109-111, 2003.

3. el-Shami K, Griffiths E and Streiff M: Nonbacterial thrombotic endocarditis in cancer patients: Pathogenesis, diagnosis and treatment. Oncologist 12: 518-523, 2007.

4. Tesselaar ME, Romijn FP, Van Der Linden IK, Prins FA, Bertina RM and Osanto S: Microparticle-associated tissue factor activity: A link between cancer and thrombosis? J Thromb Haemost 5: 520-527, 2007.

5. Masubuchi H, Maeno T, Uchida M, Kono S, Suzuki M, Takemura M, Yamaguchi A, Yamaguchi K, Kanbe M, Kitahara S, et al: A case of Trousseau syndrome caused by pulmonary adenocarcinoma that was controlled for one year and 10 months with thrombosis treatment using an EGFR tyrosine kinase inhibitor and chemotherapy. Respir Med Case Rep 15: 101-105, 2015.

6. Albright F: Case records of the Massachusetts General Hospital (case 27461). N Engl J Med 225: 789-791, 1941.

7. Wolchok JD, Herr HW and Kelly WK: Localized squamous cell carcinoma of the bladder causing hypercalcemia and inhibition of PTH secretion. Urology 51: 489-491, 1998.

8. Dvorak HF: Thrombosis and cancer. Hum Pathol 18: 275-284, 1987.

9. Wahrenbrock M, Borsig L, Le D, Varki N and Varki A: Selectin-mucin interactions as a probable molecular explanation for the association of Trousseau syndrome with mucinos adenocarcinomas. J Clin Invest 112: 853-862, 2003.

10. Shao B, Wahrenbrock MG, Yao L, David T, Coughlin SR, Xia L, Varki A and McEver RP: Carcinoma mucins trigger reciprocal activation of platelets and neutrophils in a murine model of trousseau syndrome. Blood 118: 4015-4023, 2011.

11. Carraway KL, Fregien N, Carraway KL III and Carraway CA: Tumor sialomucin complexes as tumor antigens and modulators of cellular interactions and proliferation. J Cell Sci 103: 299-307, 1992.

12. Varki NM and Varki A: Heparin inhibition of selectin-mediated interactions during the hematogenous phase of carcinoma metastasis: Rationale for clinical studies in humans. Semin Thromb Hemost 28: 53-66, 2002.

13. Tachihara M, Nikaido T, Wang X, Sato Y, Ishii T, Saito K, Sekine S, Tanino Y, Ishida T and Munakata M: Four cases of Trousseau's syndrome associated with lung adenocarcinoma. Intern Med 51: 1099-1102, 2012. 
14. Jovin TG, Boosupalli V,Zivkovic SA, Wechsler LR and Gebel JM High titers of CA-125 may be associated with recurrent ischemic strokes in patients with cancer. Neurology 64: 1944-1945, 2005.

15. Sack GH Jr, Levin J and Bell WR: Trousseau's syndrome and other manifestations of chronic disseminated coagulopathy in patients with neoplasms: Clinical, pathologic, and therapeutic features. Medicine (Baltimore) 56: 1-37, 1977.

16. Walsh-McMonagle D and Green D: Low-molecular-weight heparin in the management of Trousseau's syndrome. Cancer 80: 649-665, 1997.
17. Kumar AK, Satyan MT, Holzbeierlein J, Mirza M and Van Veldhuizen P: Leukemoid reaction and autocrine growth of bladder cancer induced by paraneoplastic production of granulocyte colony-stimulating factor-a potential neoplastic marker: A case report and review of the literature. J Med Case Rep 8: 147,2014

18. Hirasawa K, Kitamura T, Oka T and Matsushita H: Bladder tumor producing granulocy te colony-stimulating factor and parathyroid hormone-related protein. J Urol 167: 2130, 2002. 